Journal of

Cancer Research and Therapeutic Oncology

\title{
Benefit of Multimodality Image Acquisition in the Treatment Planning of Ste- reotactic Radiosurgery (SRS) of Arteriovenous Malformation (AVM)
}

Dan Ishihara, M.D, Ph.D ${ }^{1 *}$, Marco C Pinho, M.D. ${ }^{3}$, Xuejun Gu, Ph.D. ${ }^{2}$, Strahinja Stojadinovic, Ph.D. ${ }^{2}$, Hui Liu, M.S. ${ }^{2}$, Robert Timmerman, $\mathrm{MD}^{2}$

${ }^{1}$ Norton Cancer Institute, Radiation Oncology, 676 S. Floyd St., First Floor, Louisville, KY, USA 40223

${ }^{2}$ University of Texas Southwestern Medical Center, Department of Radiation Oncology, 2280 Inwood Road, Dallas, Texas, USA 75390-9303

${ }^{3}$ Department of Radiology and Advanced Imaging Research Center, UT Southwestern Medical Center, Dallas, TX, USA, 75235

*Corresponding author: Dan Ishihara, MD Ph.D. Norton Cancer Institute, Department of Radiation Oncology, 676 S. Floyd St., First Floor, Louisville, KY, USA 40223, Telephone: 502-629-4555, Fax: 502-629-4599, Email:dan.ishihara@nortonhealthcare.org

Received Date: January 09, 2019 Accepted Date: February 03, 2020 Published Date: February 05, 2020

Citation: Dan Ishihara (2020) Benefit of Multimodality Image Acquisition in the Treatment Planning of Stereotactic Radiosurgery (SRS) of Arteriovenous Malformation (AVM). J Cancer Res Therap Oncol 8: 1-10.

\begin{abstract}
Background: Digital subtraction catheter angiography (DSA) is utilized at our institution in addition to MRI for treatment planning of arteriovenous malformations (AVMs) stereotactic radio surgery (SRS). We hypothesized MRI over-predicts the size of AVM nidus and compared MRI alone versus combined imaging as input for target delineation for AVM SRS.

Methods: 90 AVMs from 78 patients treated with SRS at our institution from March 2013 to July 2017 were retrospectively analyzed. A Neuroradiologist contoured the AVM nidus from MRI alone in a blinded manner. In addition to volumetric parameters from the treatment planning software, DICOM images were exported to abstract the spatial information of the contoured volumes.

Results: Of the evaluable 81 AVMs from 69 patients, all underwent MRI and DSA. 40 patients (58\%) were male and median age was 36 (range 3-72). Median target volume from SRS treatments, AVM nidus generated by MRI alone and DSA alone were $1.33 \mathrm{~cm}^{3}, 1.13 \mathrm{~cm}^{3}$, and $1.30 \mathrm{~cm}^{3}$, respectively. The ratios of these volumes were also not significantly different, refuting our hypothesis. Interestingly, a strong agreement was found between the target and DSA-generated volume. Spatial analysis of these volumes with DICE and Jaccard Index (JI) revealed significantly greater similarity and overlap between the target and DSA-generated volume than with MRI-generated volume $(\mathrm{p}<0.0001)$.

Conclusions: SRS target AVM volume at our institution is significantly more similar to DSA-generated volume than that of MRI. This finding indicates our neurosurgeons and neurovascular surgeons rely more on the vascular data of DSA containing temporal and spatial information, albeit in a two-dimensional (2D) format when creating the target AVM nidus volume. Furthermore, a significant number of cases showed disagreement between the volumes delineated by MRI and DSA. Together, these findings support the need for multimodality image acquisition for effective treatment planning of AVM SRS.
\end{abstract}

Keywords: AVM; SRS; target delineation; DSA (digital subtraction angiography).

C2020 The Authors. Published by the JScholar under the terms of the Creative Commons Attribution License http://creativecommons.org/licenses/ by/3.0/, which permits unrestricted use, provided the original author and source are credited. 


\section{Introduction}

Brain arteriovenous malformation (AVM) is a congenital vascular anomaly where there is direct arterial to venous connections without an intervening capillary network, creating a system of feeding arteries, tangled malformation or nidus, and draining veins engorged from the high pressure. Acting as a shunt, AVM results in both a potential steal (lack of nutrition and oxygen delivered to intended brain parenchyma) as well as a high-pressure arteriovenous communication prone to spontaneous hemorrhage. Surgical resection remains the primary treatment modality for patients with AVM as it eliminates the source of steal and hemorrhage immediately. However, surgery may not be feasible in cases with AVM in deep locations and / or eloquent areas of the brain. Embolization may be used as an independent therapy, however, it is more commonly utilized prior to surgery as an adjunct (reviewed in [1]). Stereotactic Radiosurgery (SRS) is another local treatment modality that has been established as an effective treatment for brain AVM $[2,3]$. Although there is a delay in beneficial effects ranging from 6 months to 3 years, SRS is able to treat larger and deeper AVMs with more safety than surgery. In order to prevent unwanted radiation injury, accurate target delineation is essential. Because of its high temporal and spatial resolution, the gold standard technique for diagnostic and follow-up imaging of AVMs is digital subtraction catheter angiography (DSA) [4, 5]. At our institution, we routinely perform DSA on the day of the SRS procedure for improved target delineation. Following image acquisition, an interventional radiologist manually delineates the AVM nidus on the planar angiogram images; these images are stereotactically fused with the same day MR images. When stereotactically fused with MRI, the orthogonal planar DSA information can be outlined to form quadrilateral "boundaries" on each slice of the MRI. The finalized "target" nidus of the AVM is then contoured by a neurosurgeon by rounding the corners of the DSA quadrilateral using MRI signal information (Figure 1A). Such extra information from angiography allows the neurosurgeons and radiation oncologists to make judgments to partition a target volume and spare the surrounding normal brain parenchyma by incorporating anatomic and physiologic information from different image modalities.

Zhang and colleagues reported the superimposing the DSA derived AVM nidus information onto CT or MRI was an important technique to determine the precise shape of the nidus [5]. Even though the addition of angiography is time-consuming and requires multi-modality team care, our group strongly believed that this approach enabled our institution to treat the smallest possible AVM target without missing DSA identified nidus, yet achieve excellent obliteration rates with very low toxicity from the procedure. Hence, we hypothesized that the AVM nidus identified by MRI-alone would be larger than the finalized target volume. To examine this hypothesis, we designed a study to objectively compare AVM nidus treatment volumes generated with and without DSA data. Contrary to our hypothesis, data analysis revealed that our neurosurgeons and radiation oncologists have a greater degree of confidence in $2 \mathrm{D}$ vascular data to delineate the AVM nidus instead of 3D post-contrast MRI images of these AVMs. Furthermore, we found around 10\% of cases could have not been treated with confidence if DSA did not serve as a backup modality. These findings support the need for multimodality image acquisition in radio surgical treatment planning of AVM.

\section{Methods}

90 AVMs from 78 patients that were treated between March 2013 to July 2017 at the University of Texas Southwestern Medical Center using Gamma Knife ${ }^{\circledR}$ Perfection ${ }^{\mathrm{TM}}$ and/or Icon $^{\text {Tn }}$ between March 2013 to July 2017 were analyzed (Figure 1B). For the evaluable83 AVMs from 71 patients, all patients underwent MRI and interventional cerebral angiography on the day of the SRS procedure. An interventional radiologist manually delineated the AVM nidus on the planar angiogram images, which were stereotactically fused with the MR images in the radiation treatment planning software using consistent fiducials. The nidus of the AVM was then contoured by the neurosurgeon in the radiation treatment planning software and radiosurgery was performed with Gamma Knife. The brain AVM nidus was retrospectively delineated solely on MRI data by an experienced neuroradiologist M.P. at a later date. The nidus was contoured on T1-weighted post-contrast-enhanced Fast Field Echo (FFE) images. The neuroradiologist was blinded to both the DSA images and the nidus target volumes that were used for the actual treatment. Care was taken to avoid draining veins (Figure 2A) or blood products associated with the AVMs. For the purpose of this study, the authors defined the ground truths as the finalized target volume generated by the neurosurgeons and neurovascular surgeons that was used in the SRS treatment, for comparison to treatment volumes generated by the individual rater ("blinded" neuroradiologist) and interventional radiologists. Five patients that did not show a clear MRI lesion at the corresponding location of AVM identified by DSA (Figure 2B). Seven AVMs from seven patients were excluded from the analysis for the following reasons: Two had no finalized target AVM volume delineated in the treatment plans, two harbored previously treated AVMs at a different location in the brain. One had only CT 
(A)

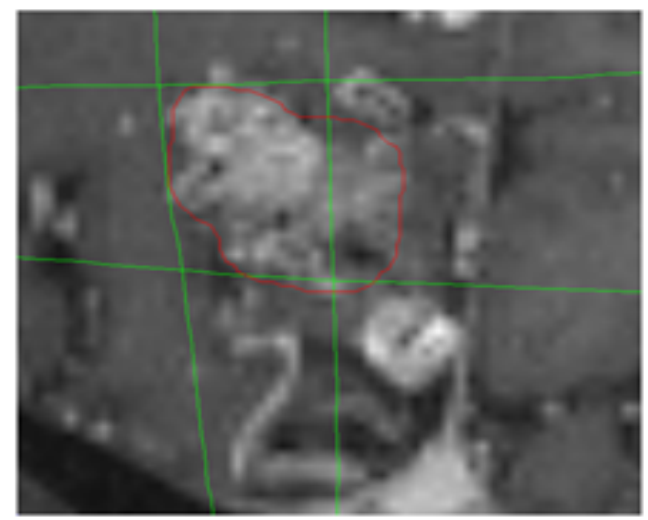

(B)

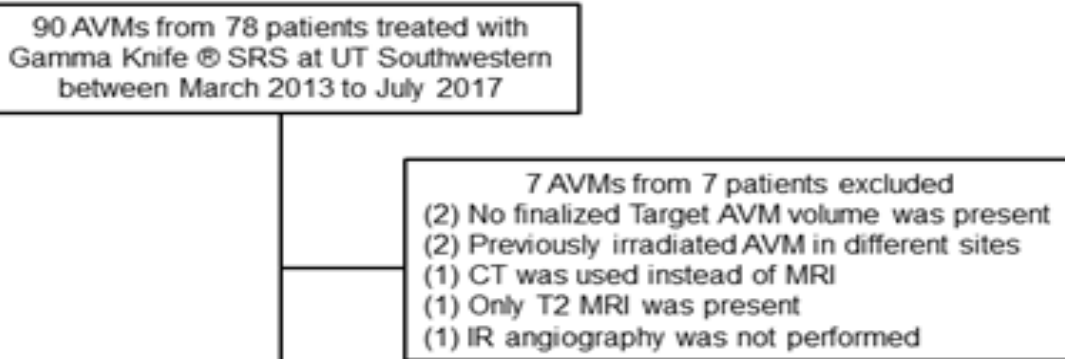

83 AVMs from 71 patients with contours
and angiography information masked

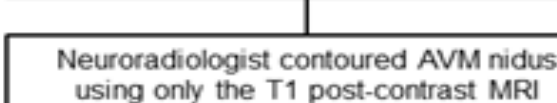

using only the T1 post-contrast MRI

2 AVMs from 2 patients excluded

(2) Inability to identify/contour the AVM nidus

Figure 1: (A) Example treatment planning where MRI abnormality appears larger than that outlined by DSA (green outline) by an interventional radiologist. The finalized SRS treatment volume (Target, red outline) was generated by a Neurosurgeon. (B) Study Scheme.

(A)

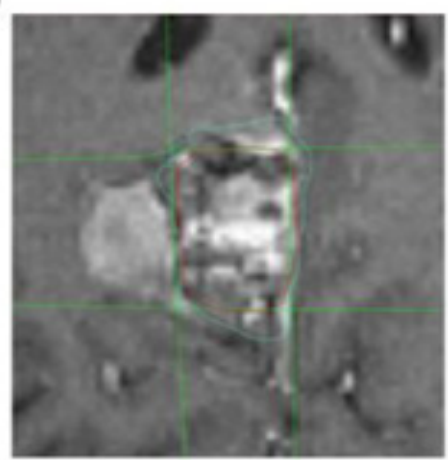

(C)

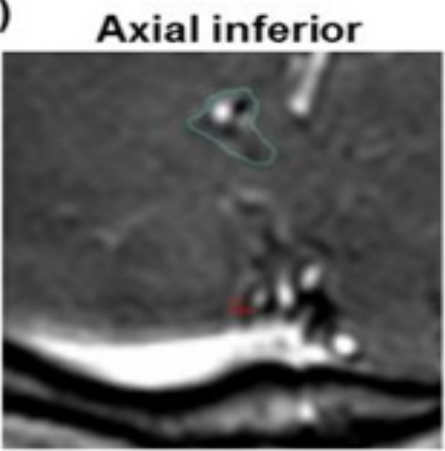

(B)
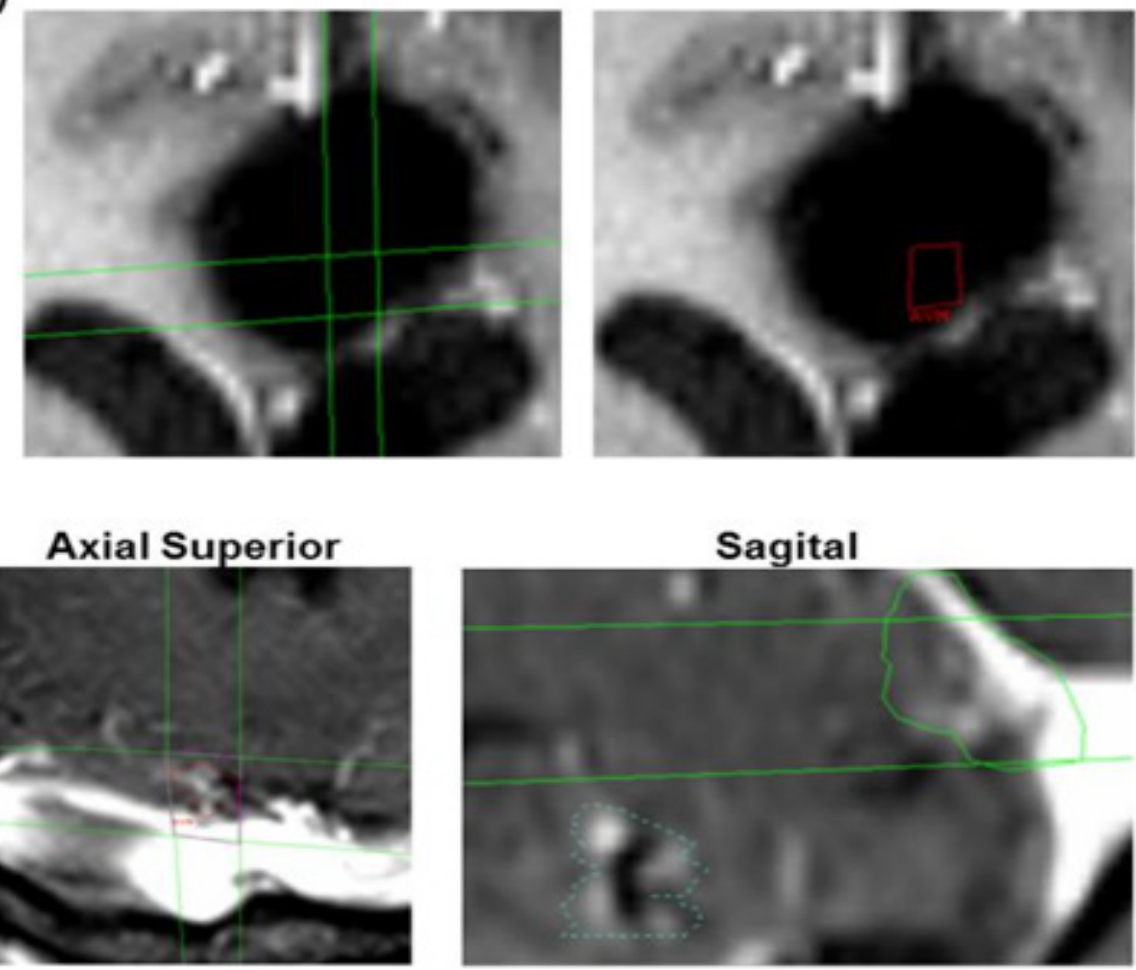

Sagital

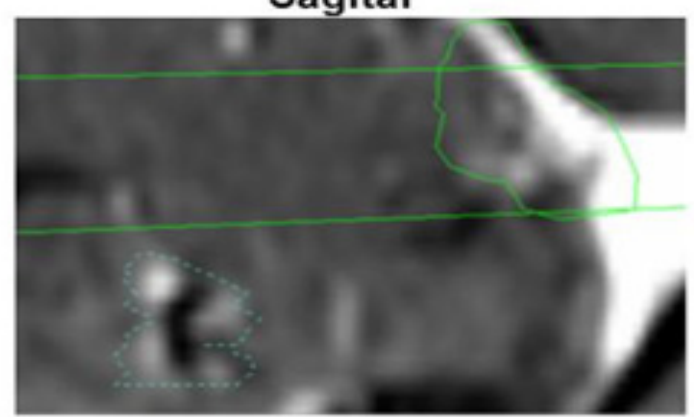

Figure 2: (A) Example treatment planning images of an AVM nidus volume generated by MRI (blue outline), AVM nidus generated by DSA alone by an Interventional Radiologist (green line) and the finalized target volume used for SRS treatment (red line) generated by a Neurosurgeon or Neurovascular surgeon. Hematoma is not included in the AVM nidus identified by both MRI and Cerebral angiography methods. (B) Example treatment planning images where MRI did not have a corresponding vessel or parenchyma within the superimposed Cerebral angiography identified area. (C) Example treatment planning images where the Neuroradiologist identified an AVM at a different location from the AVM that was identified by Angiogram and treated with SRS. 
information, one had only the T2 MRI sequence instead of the T1 post-contrast MRI and one did not include DSA. Two AVMs from two patients were not evaluable because the neuroradiologist was unable to identify the AVM nidus (Figure 2C).

The data obtained in this study are $3 \mathrm{D}$ volume sets, measured as the sum of the voxels contained within the contours from the treatment planning software of Gamma Knife ${ }^{\circledR}$ directly. Dice Similarity Coefficients (DSC) is obtained by the equation $(\mathrm{DSC}=2(\mathrm{~A} \cap \mathrm{B}) /(|\mathrm{A}|+|\mathrm{B}|))$, where $\mathrm{A}$ and $\mathrm{B}$ are the ground-truth and evaluated as segmented volumes, respectively[6]. The Jaccard index (JI) is a measure of the overlap that A and B share with their attributes, which is defined as $\mathrm{JI}=(\mathrm{A} \cap \mathrm{B}) /(\mathrm{AUB})[7]$. Both the values of DSC and JI ranges from 0 to 1 , indicating no spatial similarity and overlap, respectively. DSC and JI of the target, MRI, and DSA generated volumes were independently calculated algorithm from the DICOM structured data sets.

\section{Statistical Analysis}

All statistical analyses, including the matched t-test analysis, one-way ANOVA, corresponding figures and tables and Bland-Altman Plots $[8,9]$ were created using Graph Pad 5 software. Bland-Altman plot calculates the mean difference between two methods of measurement (MRI and DSA in this case). The graphic display of agreement is presented with the mean of two measures in the $\mathrm{x}$-axis and the difference between two measures in the y-axis. The $95 \%$ limits of agreement (LoA) were computed and depicted by 1.96 standard deviations that encompass above and below the line of agreement between the two methods of measurement.

\section{Results}

\section{Patient Demographics and AVM Characteristics}

81 AVMs from 69 patients treated with Gamma Knife 'SRS at the University of Texas Southwestern Medical Center between March 2013 to July 2017 were analyzed. 40 patients (58\%) were male and 29 patients (42\%) were female. The median age was 36 (range 3-72). 58 AVMs (71.6\%) were in the cerebrum, 11 AVMs (13.6\%)in the cerebellum, seven AVMs (8.6\%) in the brainstem, four AVMs (4.9\%) intraventricular and one AVM (1.2\%) near the auricular nerve. The median AVM nidus volume of finalized target volume used in SRS targeting, AVM nidus generated by MRI alone (MRI) and AVM nidus generated by cerebral angiography (DSA) were $1.33 \mathrm{~cm}^{3}$ (range $0.05-14.5 \mathrm{~cm}^{3}$, mean $2.47 \mathrm{~cm}^{3}$ ), $1.13 \mathrm{~cm}^{3}$ (range $0.27-15.1 \mathrm{~cm}^{3}$, mean $2.63 \mathrm{~cm}^{3}$ ) and $1.30 \mathrm{~cm}^{3}$ (range $0.05-16.6 \mathrm{~cm}^{3}$, mean $2.97 \mathrm{~cm}^{3}$ ), respective- ly. To assess the change in volume of AVM nidus target by the use of DSA, the raw volumetric segmentation data were paired to determine the ratio of the MRI alone to the finalized target volume of each SRS treatment plan. Contrary to our prediction, only about half the cases (44 of the 81 AVMs, 54\%) showed the MRI-generated volume was larger than the targeted volume (Figures $3 \mathrm{~A}$ and3B). As such, the volume ratio calculated by dividing the MRI-generated volume with the target volume was also not statistically significant (mean 1.67 and median 1.04). Paired t-test analysis was not statistically significantly different between the two volumes nor was the comparison with DSA generated volume (Figure 3C). One-way ANOVA comparison of the target, MRI-generated and DSA-generated volumes also did not reveal any statistically significant difference among the three volumes (Table1).

This was a surprise as we hypothesized that combining two imaging modalities of MRI and DSA result in a smaller target volume as the overlaid images appear to shrink the target boundary as depicted in figure 1A. It became apparent, however, that the finalized target volume appeared more closely related to the DSA-generated volume than with the MRI-generated volume. In fact, Bland-Altman plot evaluation of the agreement between two methods revealed that the $95 \%$ limits of agreement are tighter in the plot between the target and DSA-generated volumes than that between target and MRI-generated volumes (Figure 4), suggesting greater agreement between the target and DSA-generated volumes. Bland-Altman plot provides a graphical display of the agreement between the two methods of measurements of the AVM volume [2,3] but there is no test statistic to compare two Bland Altman plots with mutual measurement (in this case, the target volume) [10]. Moreover, both plots have four outliers beyond the $95 \%$ limits of agreement, which is difficult to interpret. Hence, we decided to analyze the segmentation data of each contoured AVM did with 3D coordinates instead of treating each AVM nidus as lumped "volume" without spatial relationship.

To make judgments and draw conclusions about these $3 \mathrm{D}$ structures, we compared the spatial relationships of these volumes using two common metrics: Dice Similarity Coefficient (DSC)[6] and Jaccard Index (JI)[7]. The volumetric DSC is defined as the intersection of two masks normalized to their mean volume, where $\mathbf{A}$ and $\mathbf{B}$ are the masks and $\mathbf{N}$ is an operator yielding the number of voxels (Figure $5 \mathrm{~B}$ ) whereas $\mathrm{JI}$ is defined as the intersection between $\mathrm{A}$ and $\mathrm{B}$, divided by their union. The range of DSC and JI is zero to one, where zero indicates no overlap and one indicates exact overlap. Matched pair analysis of DSC 
showed that the volumetric overlap between the finalized target and DSA-generated volume was greater ( $p$-value $<0.0001)$ than that between the finalized target and the MRI-generated volume (Figure 5C, Table 2).

Similarly, matched-pair analysis of JI showed that the volumetric overlap between the finalized target and DSA-generated volume was greater than with MRI-generated volume, with statistical significance (p-value $<0.0001$ ) (Figure 5D, Table 3 ).
Notably, when five cases of AVM lesions that did not harbor corresponding MRI contour were excluded from the analysis and subjected to matched pair-analysis, both the DSC and JI were still statically significantly higher between target volume and DSA-identified volume compared to that between target volume and MRI-alone-identified volume, revealing the higher degree of spatial agreement or congruence of the target and DSA-generated volumes.
(A)

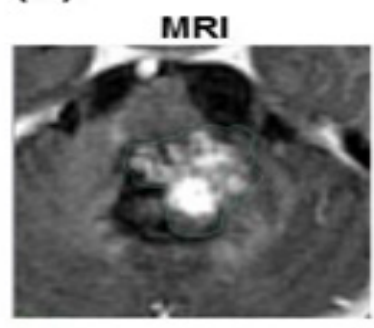

(B)

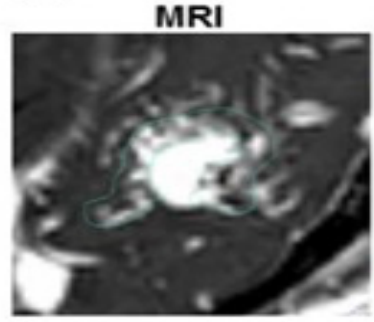

DSA

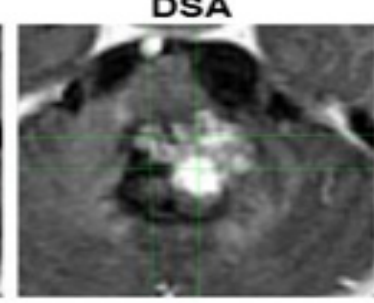

DSA

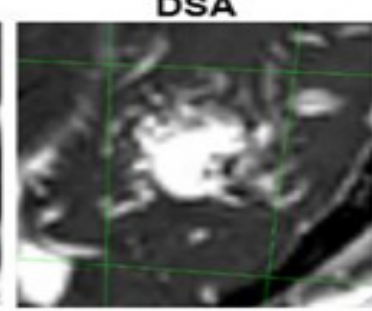

(C)

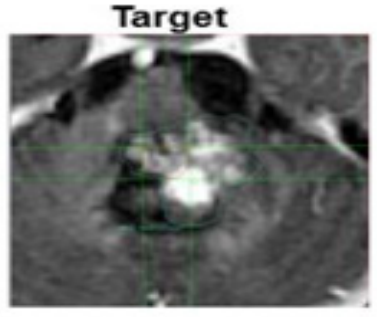

Target

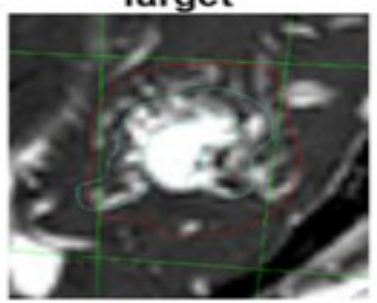

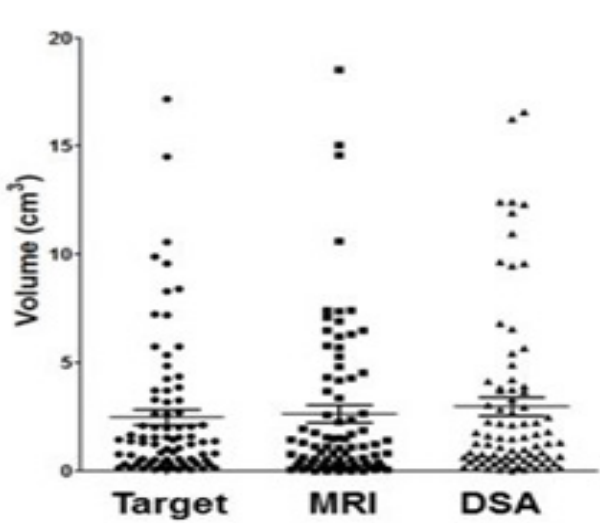

Target MRI DSA

Figure 3: (A) Example treatment planning where the AVM nidus volume generated by MRI alone by a neuroradiologist (MRI, blue outline) was larger than that generated by DSA alone by an interventional radiologist superimposed on MRI (green outline). The finalized SRS treatment volume (Target, red outline) generated by a Neurosurgeon. (B) Example treatment planning images where the DSA volume was larger than that generated from MRI alone. (C) Volume scatter plot showing the distribution of finalized SRS treatment volume (Target), AVM nidus identified by MRI alone (MRI) and digital subtraction angiography (DSA).

\begin{tabular}{|l|l|l|l|}
\hline One-way analysis of variance & & & \\
\hline P value & 0.662 & & \\
\hline P value summary & ns & & \\
\hline Are means significantly different? $(\mathrm{P}<0.05)$ & No & & \\
\hline Number of groups & 3 & & \\
\hline F & 0.4132 & & \\
\hline R squared & 0.003432 & & \\
\hline Bartlett's test for equal variances & & & \\
\hline Bartlett's statistic (corrected) & 2.151 & & \\
\hline P value & 0.3411 & & \\
\hline P value summary & ns & & \\
\hline Do the variances differ signif. $(\mathrm{P}<0.05)$ & No & & \\
\hline ANOVA Table & SS & df & MS \\
\hline Treatment (between columns) & 10.78 & 2 & 5.388 \\
\hline Residual (within columns) & 3129 & 240 & 13.04 \\
\hline Total & 3140 & 242 & \\
\hline
\end{tabular}

Table 1: One-way ANOVA comparison of the target, MRI-generated and DSA-generated volumes Abbreviations:df: degrees of freedom, MS: mean squares, ns not significant, SS: sum-of-squares. 


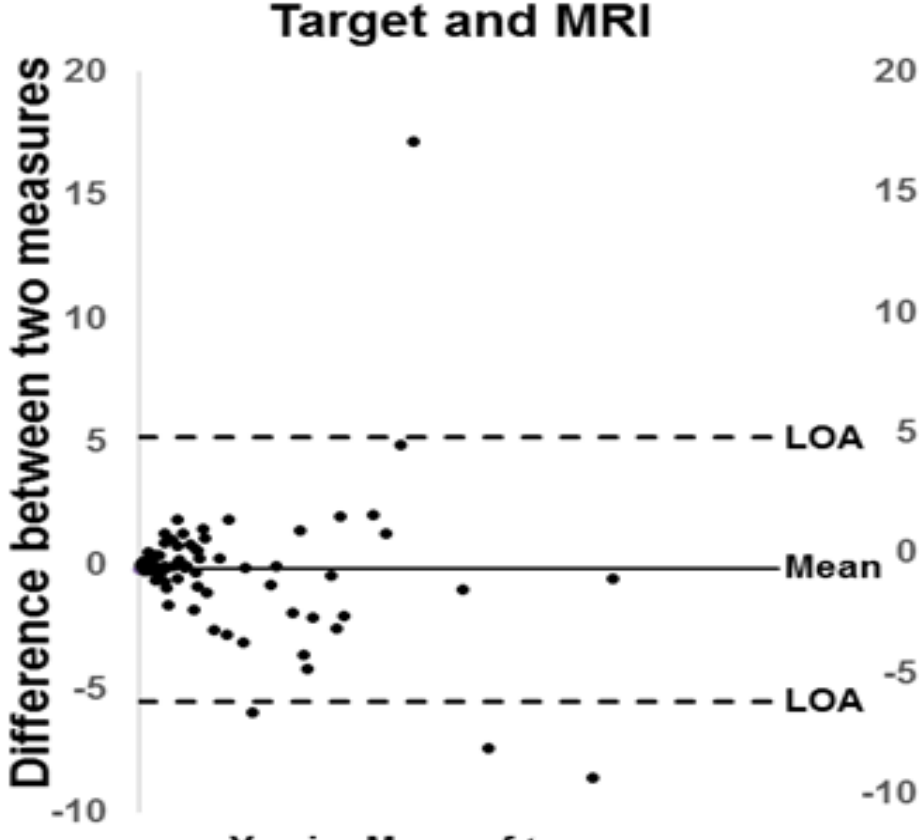

Y-axis: Mean of two measures
Target and DSA

20

15

10
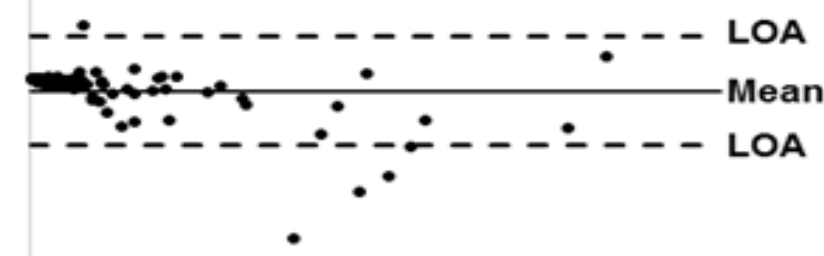

Figure 4: Bland-Altman plots of the contoured AVM volumes that assess the agreement between the two measures on an individual level. The 95\% limits of agreement (LOA) is depicted by the dotted line that represents the 1.96 standard deviation from the mean of the measurements by two methods. Finalized SRS treatment volume (Target), AVM nidus identified by MRI alone (MRI) and AVM nidus identified by digitally subtraction angiography (DSA).

(A)

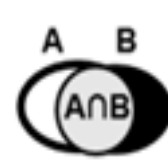

(B)
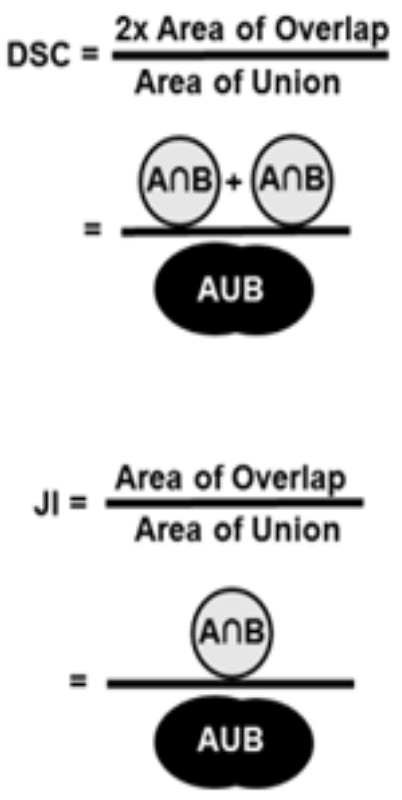

(D)

(C)
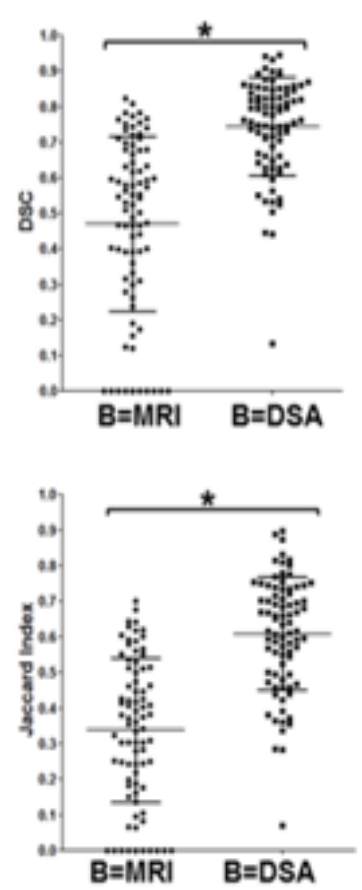

Figure 5: (A) Graphic representation of two overlapping volumes $A$ and $B$. (B) Mathematical equation and graphic representation of the Dice Similarity Coefficient (DSC) and Jaccard Index (JI). (C) Scatter plot of DSC of finalized treatment volume (Target) and AVM nidus identified by MRI alone (MRI), noted as $\mathrm{B}=\mathrm{MRI}$, compared to target and AVM nidus identified by digital subtraction angiography (DSA), noted as B=DSA. (D) Scatter plot of JI of Target and MRI, noted as B=MRI, compared to Target and DSA noted as B=DSA. 


\begin{tabular}{|l|l|l|}
\hline Table Analyzed: DSC & Target:MRI & Target:DSA \\
\hline Number of values & 81 & 81 \\
\hline Minimum & 0 & 0.1327 \\
\hline $25 \%$ Percentile & 0.3129 & 0.6613 \\
\hline Median & 0.5297 & 0.7763 \\
\hline $75 \%$ Percentile & 0.6778 & 0.8467 \\
\hline Maximum & 0.824 & 0.9456 \\
\hline Mean & 0.4702 & 0.7437 \\
\hline Std. Deviation & 0.2465 & 0.1377 \\
\hline Std. Error & 0.02739 & 0.0153 \\
\hline Lower 95\% CI & 0.4157 & 0.7133 \\
\hline Upper 95\% CI & 0.5247 & 0.7742 \\
\hline Paired t test & & \\
\hline P value & $<0.0001$ & \\
\hline Are means signif. different? $(\mathrm{P}<0.05)$ & Yes & \\
\hline One- or two-tailed P value? & Two-tailed & \\
\hline $\mathrm{t}$, df & $\mathrm{t}=8.260 \mathrm{df}=80$ & \\
\hline Number of pairs & 81 & \\
\hline
\end{tabular}

Table 2Matched pair analysis of Dice Similarity Coefficients(DSC) between the finalized target and DSA-generated volume compared to DSC between the finalized target and the MRI-generated volume.

Abbreviation: df: degrees of freedom, DSC: Dice Similarity Coefficients

\begin{tabular}{|l|l|l|}
\hline Table Analyzed: Jaccard Index & Target:MRI & Target:DSA \\
\hline Number of values & 81 & 81 \\
\hline Minimum & 0 & 0.07106 \\
\hline $25 \%$ Percentile & 0.1854 & 0.494 \\
\hline Median & 0.3603 & 0.6344 \\
\hline $75 \%$ Percentile & 0.5127 & 0.7342 \\
\hline Maximum & 0.7007 & 0.8969 \\
\hline Mean & 0.3391 & 0.609 \\
\hline Std. Deviation & 0.202 & 0.159 \\
\hline Std. Error & 0.02245 & 0.01766 \\
\hline Lower 95\% CI & 0.2945 & 0.5738 \\
\hline Upper 95\% CI & 0.3838 & 0.6441 \\
\hline Paired t test & & \\
\hline P value & $<0.0001$ & \\
\hline Are means signif. different? $(\mathrm{P}<0.05)$ & Yes & \\
\hline One- or two-tailed P value? & Two-tailed & \\
\hline$t$, df & $\mathrm{t}=9.519 \mathrm{df}=80$ & \\
\hline Number of pairs & 81 & \\
\hline
\end{tabular}

Table 3Matched pair analysis of Jaccard Index between the finalized target and DSA-generated volume compared to DSC between the finalized target and the MRI-generated volume.

Abbreviation: df: degrees of freedom 


\section{Discussion}

The gold standard technique for the diagnosis and follow-up imaging of AVMs remains digital subtraction catheter-derived DSA. At our institution, we employ stereotactic targeting provided by the reference head frame during image acquisition of MRI and DSA to superimpose the 2D image of DSA generated volume to MRI to identify the nidus configuration. In 1993, Guo et al. in 1993 reported the feasibility of delineating an AVM on MRI and compared this AVM volume to that outlined in angiogram from six patients with seven AVMs [11]. In contrast to our initial hypothesis, Guo et al. reported that the estimated volumes of the AVM nidi were larger with the conventional stereotactic angiograms than that identified by MRI. While the early works by Guo and colleagues are very insightful, they derived their conclusions from a very small sample size compared to our present study. Moreover, the authors noted the difficulty in imaging smaller AVMs with MRI, which echoes the technological advances in MRI since the early 1990s with greater ability to image smaller AVMs with finer details. Higher field strength magnets and other hardware and software advances have most certainly improved both the accuracy and precision of MR image acquisition for an intracranial AVM.

While we hypothesized that the use of DSA enabled physicians to delineate a smaller AVM target in conjunction with MRI, our result showed that the use of angiogram decreased the target volume in only about half the cases when compared with MRI alone. Nonetheless, the present analysis revealed two interesting findings. The first finding is that the neurosurgeons and neurovascular surgeons at our institution rely on DSA data when creating the target AVM nidus volume more than on MRI-based data. This finding was surprising at first since the Gamma Knife treatment planning and treatment delivery of SRS is based on MRI. In retrospect, however, this observation makes sense, as our neurosurgeons and neurovascular surgeons rely on the temporal and spatial resolution provided by DSA to more accurately delineate the AVM nidus. Despite being a 2D image, which needs to be superimposed onto the treatment planning MRI, the 2D information obtained from the DSA contains crucial kinetic information about filling of the nidus and associated draining veins, as opposed to the static information provided by the T1-weighted, contrast-enhanced MRI. The relatively low-resolution MRI images provide a suboptimal distinction between the nidus, nearby draining veins and other abnormalities, including sub acute blood products and enhancing scar tissue (in AVMs with prior hemorrhage).

JScholar Publishers
A second finding from our study is that the choice of volume-based comparison metrics is critical as each yield different information and must be considered in the appropriate context. Spatial overlap measures such as the DSC and JI have been broadly adopted in the field of segmentation analysis [12]. Precision and accuracy are crucial concepts in target delineation, as one without the other does not allow precise treatment delivery that leads to greater sparing of normal brain parenchyma. Our analysis indicated that in a non-insignificant number of cases (11 out of 90 AVMs, 12\% in our cohort) either MRI or Angiography could not be performed or were not in agreement, as in the case depicted in Figure 2B where an AVM nidus was identified in what appears to be a cavity on MRI. In these circumstances, a practitioner without a second imaging modality will be at loss identifying targets, unable to deliver treatment with confidence. In another instance, a very small AVM $(<1 c c)$ near the confluence of sinuses was not visualized well by MRI. Variables such as AVM volume, location in relation to draining vein/sinus/confluence, evidence of prior hemorrhage or the ratio between nidus/ draining veins were considered but the number of patients was too small to make any inference. Of note, it is unlikely that the superimposition of the images was compromised since the stereotactic head frame serves as $3 \mathrm{D}$ coordinates for the fusion of images.

Various techniques have been proposed to delineate complex AVM nidus configuration. A case report from the University of Massachusetts reported a multimodality image acquisition technique to identify a micro AVM that was too small to be visualized by conventional methods [13]. Investigators reported acquisition of high-resolution cone-beam CT using an angiographic c-arm system to provide image quality comparable to that of multi detector CT. They registered MRI, non-contrast CT and cone-beam CT data to the radiation planning software and successfully obliterated the micro AVM. MR Angiographic techniques such as $3 \mathrm{D}$ phase-contrast and contrast-enhanced 3D time-of-flight technique are now widely employed and allow selective evaluation of fast-flowing blood in the vascular system with optional gradient-moment nulling. These MRI based techniques can depict the anatomic features of cerebral AVM, however, they provide only static information [14]. Other sequences, such as susceptibility-weighted imaging (SWI) allows the visualization of large and small veins in the brain without the use of an exogenous contrast, through the use of magnitude or phase images obtained with the three-dimensional, velocity-compensated, gradient-echo sequence. Black blood MRI sequences are able to selectively suppress flowing blood [15] and may also have a role to identify the nidus more accurately and precisely. More 
recently, contrast-enhanced, time-resolved MRI angiography has been proposed as a non-invasive alternative to provide temporal information close to that obtained with DSA [16]. Unfortunately, these advanced MRI sequences usually require longer acquisition times and are often not feasible in patients with a stereotactic frame in position. In the future, frame-less SRS, such as Gamma Knife ${ }^{\circledR}$ Icon $^{\text {mm }}$ or fiducial marker implanted into the patient's skull before angiography [17], could be coupled with contrast-enhanced, time-resolved MRI angiography to delineate AVM nidus.

In our study, the neuro radiologist felt that some AVMs were particularly difficult to reliably identify on the post-contrast T1-weighted images, including lesions associated with prominent normal enhancing structures (such as the choroid plexus), lesions closely related to dural sinuses and those surrounded by evolving blood products or scar tissue from prior hemorrhage. For these types of lesions, the information obtained from DSA is particularly invaluable. Despite its superior temporal and spatial resolution, cerebral angiography has significant disadvantages, including its invasive nature requiring an arterial puncture and non-insignificant complication rate [18-20]. It is likely that the use of MRI techniques more specific for arterial flow (versus post-contrast T1-weighted images) would improve target delineation and eventually obviate the need to perform DSA. However, these new acquisition techniques require prospective comparison and validation with an AVM nidus delineated by the gold standard DSA.

\section{Conclusions}

Treatment SRS AVM volumes are significantly more similar to catheter DSA-generated volumes than to MRI-generated volumes when compared using spatial overlap measures at our institution. These results demonstrate that our neurosurgeons and radiation oncologists have a greater degree of confidence on 2D, high temporal and spatial resolution of the vascular data than on 3D post-contrast MRI images of these AVMs when deciding final treatment plans. Furthermore, our data suggest that about $10 \%$ of cases would have not been treated if DSA was not performed and served as a backup modality. These findings support the need of multimodality image acquisition to compensate for the uncertainty in the precision and accuracy of inference of 3D geometries of AVM nidus from T1-weighted post contrast MRI alone. Given the invasive nature and non-insignificant potential complications of catheter angiography, future studies exploring the accuracy of other more advanced MRI techniques are warranted.

\section{References}

1. Lunsford LD, Niranjan A, Kondziolka D, Sirin S, Flickinger JC (2008) Arteriovenous malformation radiosurgery: a twenty-year perspective. Clin Neurosurg 55:108-119.

2. Flickinger JC, Kondziolka D, Maitz AH, Lunsford LD (2002) An analysis of the dose-response for arteriovenous malformation radiosurgery and other factors affecting obliteration. Radiother Oncol 63: 347-354.

3. Shin M, Kawamoto S, Kurita H, Tago M, Sasaki T, Morita A, Ueki K, Kirino T (2002) Retrospective analysis of a 10 -year experience of stereotactic radiosurgery for arteriovenous malformations in children and adolescents. J Neurosurg 97: 779-784.

4. Coste E, Gibon D, Lecercq X, Verdonck B, Vasseur C, Rousseau J (2001) 3D reconstruction of the encapsulating contour of arteriovenous malformations for radiosurgery using digital subtraction angiography. Int J Radiat Onco 150: 247-255.

5. Zhang X-Q, Shirato H, Aoyama H, Ushikoshi S, Nishioka T, Zhang DZ, Miyasaka K (2003) Clinical significance of 3D reconstruction of arteriovenous malformation using digital subtraction angiography and its modification with CT information in stereotactic radiosurgery. Int J Radiat Onco 157: 1392-1399.

6. Dice L (1945) Measures of the amount of ecologic association between species (1945) Ecology 26: 297-302.

7. Jaccard P (1908) Nouvelles recherches sur la distribution florale. Bulletin de la SocieteVaudoise des Sciences Naturelles 44: 223-270.

8. Altman DG and Bland JM (1983) Measurement in medicine: the analysis of method comparison studies. The Statistician 32: 307-317.

9. Bland JM and Altman DG (1986) Statistical methods for assessing agreement between two methods of clinical measurement. Lancet 1: 307-310.

10. Hanneman SK (2008) Design, analysis, and interpretation of method-comparison studies. AACN Adv Crit Care 19: 223-234.

11. Guo WY, Nordell B, Karlsson B, Soderman M, Lindqvist M, et al (1993) Target delineation in radiosurgery for cerebral arteriovenous malformations. Assessment of the value of stereo taxic MR imaging and MR angiography. Acta Radiol 34: 457463.

12. Zijdenbos A, Dawant B, Margolin A, Palmer AC (1994) Morphometric analysis of white matter lesions in MR images: Method and validation. IEEE Trans Med Imag13: 716-724.

13. van der Bom IM, Gounis MJ, Ding L, Kuhn AL, Goff D, Puri AS, Wakhloo AK (2014) Target delineation for radiosur- 
gery of a small brain arteriovenous malformation using high-resolution contrast-enhanced cone-beam CT. J Neuro interv Surg 6:e34.

14. Marks MP, Pelc MJ, Ross MR, Enzmann DR (1992) Determination of cerebral blood flow with a phase-contrast cine MR imaging technique: evaluation of normal subjects and patients with arteriovenous malformation. Radiology 182: 467-476.

15. Etminan N, Brown RD Jr, Beseoglu K, Juvela S, Raymond J, Morita A, et al. (2015) The unruptured intracranial aneurysm treatment score: a multidisciplinary consensus. Neurology 85: 881-889.

16. Oleaga L, Dalal SS, Weigele JB, Hurst RW, Lee J, Voorhees A, et al. (2010) The role of time-resolved 3D contrast-enhanced MR angiography in the assessment and grading of cerebral arteriovenous malformations. Eur J Radio 174: e117-121.

17. Lu XQ, Mahadevan A, Mathiowitz G, Lin PJ, Thomas A, Kasper EM, et al. (2012) Frameless angiogram-based stereotactic radiosurgery for treatment of arteriovenous malformations. Int J Radiat Oncol 84: 274-282.

18. Fung E, Ganesan V, Cox TS, Chong WK, Saunders DE (2005) Complication rates of diagnostic cerebral arteriography in children. Pediatr Radiol 35: 1174-1177.

19. Abu Rahma AF, Hayes JD, Deel JT, Abu-Halimah S, Mullins BB, Habib JH, et al. (2006) Complications of diagnostic carotid/cerebral arteriography when performed by a vascular surgeon. Vasc Endovascular Surg 40: 189-195.

20. Dawkins AA, Evans AL, Wattam J, Romanowski CA, Connolly DJ, Hodgson TJ, et al. (2007) Complications of cerebral angiography: a prospective analysis of 2,924 consecutive procedures. Neuroradiology 49: 753-759.
Submit your manuscript to a JScholar journal and benefit from:

ब Convenient online submission

- Rigorous peer review

I Immediate publication on acceptance

- Open access: articles freely available online

ब High visibility within the field

I Better discount for your subsequent articles

Submit your manuscript at http://www.jscholaronline.org/submit-manuscript.php 